\title{
Status of the GERDA Experiment
}

\author{
Andrea Lazzaro* on behalf of the GERDA collaboration \\ Physik-Department and Excellence Cluster Universe, Technische Universität München, \\ Germany \\ E-mail: andrea.lazzaro@tum.de
}

The GERDA experiment searches for neutrinoless double beta decay in ${ }^{76} \mathrm{Ge}$. In its first phase, GERDA collected $21.6 \mathrm{~kg} \cdot \mathrm{yr}$ of exposure with a background index (B.I.) of $0.01 \mathrm{cts} /(\mathrm{keV} \cdot \mathrm{kg} \cdot \mathrm{yr})$. No signal was observed and a lower limit for the half-life of the process has been set to $2.1 \cdot 10^{25} \mathrm{yr}(90 \%$ C.L.).

The apparatus has been upgraded to the Phase II configuration with 30 additional custom made BEGe detectors with better energy resolution and better pulse shape discrimination potential. For a further reduction of the B.I. light detectors have been installed to veto background events which produce scintillation light in the liquid argon surrounding the Ge detectors.

GERDA Phase II is currently taking data with 37 HPGe detectors enriched in ${ }^{76} \mathrm{Ge}$ for a total mass of $35 \mathrm{~kg}$. The goal of Phase II is to increase the half-life sensitivity to the order of $10^{26} \mathrm{yr}$, this will require $100 \mathrm{~kg} \cdot \mathrm{yr}$ exposure with a B.I. of $10^{-3} \mathrm{cts} /(\mathrm{keV} \cdot \mathrm{kg} \cdot \mathrm{yr})$.

XIII International Conference on Heavy Quarks and Leptons

22-27 May, 2016

Blacksburg, Virginia, USA

${ }^{*}$ Speaker. 


\section{Neutrinoless double beta decay}

The double beta decay (1.1) is a second order process that has been observed in 11 even-even nuclei for which the single beta decay is energetically forbidden $[1,2]$. The half-life of this process have been measured for several isotopes in the range from $10^{19}$ to $10^{24}$ years. Many extensions of the standard model of particle physics predict the existence of a channel in which no neutrinos would be produced. The neutrinoless double beta decay (1.2), forbidden by the standard model, would violate the lepton number conservation by two units and its observation would imply that neutrinos are Majorana particles [3]. In the assumption that the decay is mainly driven by the exchange of light Majorana neutrinos, it is possible to relate the half-life of the $0 v \beta \beta$ process to the scale of the neutrino masses (1.3).

$$
\begin{gathered}
2 v \beta \beta:(A, Z) \rightarrow(A, Z+2)+2 e^{-}+2 \bar{v}_{e} \\
0 v \beta \beta:(A, Z) \rightarrow(A, Z+2)+2 e^{-} \\
\frac{1}{T^{0 v}} \propto m_{e e} \equiv\left|\sum U_{e i}^{2} m_{i}\right|
\end{gathered}
$$

\section{The GERDA experiment}

The GERDA experiment searches for $0 v \beta \beta$ decay of ${ }^{76} \mathrm{Ge}$. The electrons' energy is measured with high purity germanium detectors (HPGe) enriched in ${ }^{76} \mathrm{Ge}$. The enrichment increases the concentration of the isotope to $\sim 86 \%$. The HPGe detectors provide an extremely high energy resolution $\left(0.2 \%\right.$ FWHM) at the Q-value of the process $\left(Q_{\beta \beta}=2039 \mathrm{keV}\right)$. The energy resolution of the detectors is extremely important, since the experimental signature of the $0 v \beta \beta$ is a peak at the $Q_{\beta \beta}$. The $0 v \beta \beta$ counts must be distinguished from other events coming from backgrounds as for example the intrinsic background of the $2 v \beta \beta$, in which a fraction of energy is carried away by the neutrinos.

Another advantage of solid state detectors is the high detection efficiency. Only a small fraction of the outer volume ( $\sim 10 \%$ of the enriched $\mathrm{Ge})$ is not active, and shields the bulk volume from external sources of $\alpha$ and $\beta$. The efficiency to detect the full energy of 2 electrons is also very high $(90-92 \%)$ thanks to the short distance these can move in germanium before thermalization $(1-2 \mathrm{~mm})$.

With specific pulse shape analysis (PSA) it is possible to gather information about the topology and the location of the interactions in the HPGe detectors. The background can hence be reduced selecting the signals related to energy deposited in a single site in the detector's bulk volume (SSE), as is expected from a $0 v \beta \beta$ decay.

The GERDA (Germanium Detector Array) experiment is located at the Laboratori Nazionali del Gran Sasso (LNGS) of INFN. Several HPGe detectors are mounted in low mass holders realized with ultra-low activity materials (Cu, Si and Teflon) and immersed in $65 \mathrm{~m}^{3}$ of liquid argon (LAr), see Fig. 1. The LAr bath serves two purposes: cooling the crystals to the operational temperature and shielding them from external radiation. The LAr cryostat is surrounded by $590 \mathrm{~m}^{3}$ of ultra-pure 
water which besides providing a further shield against $\gamma$ 's and neutrons, is instrumented with 66 PMTs for the detection of muons induced Cherenkov light. From the clean room above the water tank is possible to access the cryostat for the insertion of the detectors' array and of the calibration sources. More details about the experimental setup can be found in [4]

Two types of detectors are employed in the GERDA experiment (Fig. 2). The semi-coaxial detectors are p-type HPGe diodes with the read out p+ contact on the surface of a hole bored along the axis. The GERDA semi-coaxial detectors weigh about $2-3 \mathrm{~kg}$. During Phase I 8 enriched semicoaxial detectors have been used for a total mass of $17.7 \mathrm{~kg}$. In Phase II 7 of these detectors (for a total mass of $15.8 \mathrm{~kg}$ ) are operated in the array.

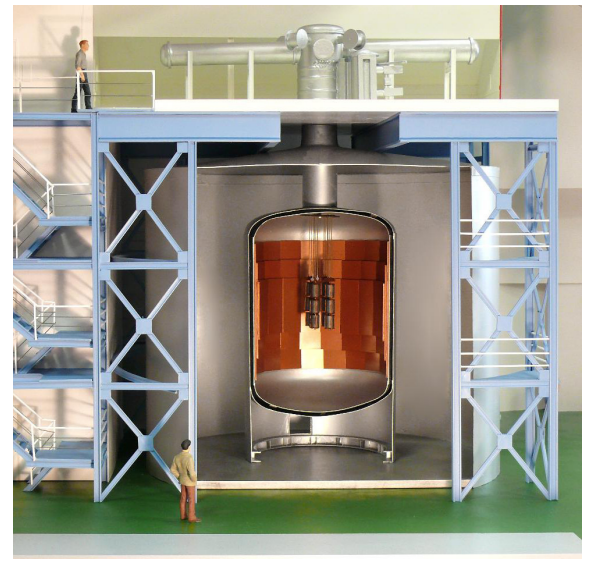

Figure 1: Model of the GERDA design. Visible are the water tank, the LAr cryostat with the ultra-clean copper shield on the wall and the lock system on the top.

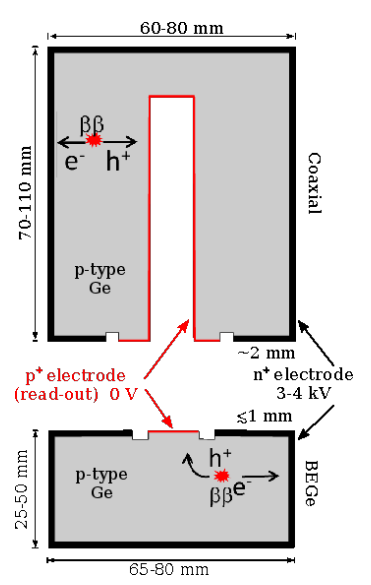

Figure 2: Description of semi-coaxial and BEGe detectors.

The other type of detectors used in GERDA is the Broad Energy Germanium (BEGe), see the bottom part of Fig. 2. These are custom made detectors with, on average, a mass of $0.7 \mathrm{~kg}$. They have smaller capacitance and better energy resolution with respect to the semi-coaxial ones. Moreover with the enhanced pulse shape discrimination (PSD) capabilities of the BEGe detectors it is possible to select with high efficiency (acceptance $\sim 90 \%$ ) $0 v \beta \beta$-like signals due to single site bulk events (SSE) against multi-site events (MSE) and surface events [5]. The $\gamma$ background in the ROI is reduced to less than a half, almost to its SSE component, i.e. single Compton scattering [6]. The surface background can be reduced by more than one order of magnitude, removing the contributions of external $\alpha$ (acceptance $<8 \%$ at $95 \%$ C.I.) and $\beta$ (acc. $<1.6 \%$ at $95 \%$ C.I.) emitters [7]. The first string with 5 BEGe detectors $(3.63 \mathrm{~kg})$ has been operated during the second part of GERDA Phase I; in Phase II 30 BEGe detectors $(20.0 \mathrm{~kg}$ ) constitute more than half of the target mass.

\section{Gerda Phase I}

Between November 2011 and May 2013 GeRDA Phase I collected $21.6 \mathrm{~kg} \cdot \mathrm{yr}$ of exposure. The array was constituted by 3 strings of 3 semi-coaxial detectors plus a string with 5 BEGe 
detectors deployed in June 2012. Some of the diodes were not considered for the $0 v \beta \beta$ analysis: one of the semi-coaxial was not isotopically enriched; two of the semi-coaxial detectors suffered high leakage current and had to be switched off; one BEGe channel showed frequent fluctuations of the gain. The total mass considered for the analysis was $17.6 \mathrm{~kg}$.

The insertion of the BEGe string perturbed the system and caused an increase in the background count-rate that lasted for several weeks. The exposure was therefore divided in three parts: the silver data set with the semi-coaxial data with higher background index, the golden data set with the remaining semi-coaxial data, and the BEGe data set which has specific features in terms of PSD, energy resolution and background composition. The golden data set has an exposure of $17.9 \mathrm{~kg} \cdot \mathrm{yr}$ and a background index of $11_{-2}^{+2} \cdot 10^{-3} \mathrm{cts} /(\mathrm{keV} \cdot \mathrm{kg} \cdot \mathrm{yr})$. The silver data set has an exposure of $1.3 \mathrm{~kg} \cdot \mathrm{yr}$ and a background index of $30_{-9}^{+11} \cdot 10^{-3} \mathrm{cts} /(\mathrm{keV} \cdot \mathrm{kg} \cdot \mathrm{yr})$. The BEGe data set has an exposure of $2.4 \mathrm{~kg} \cdot \mathrm{yr}$ and a background index of $5_{-3}^{+4} \cdot 10^{-3} \mathrm{cts} /(\mathrm{keV} \cdot \mathrm{kg} \cdot \mathrm{yr})$.

The detectors' performances (gain, energy resolution and PSD) have been monitored with (bi)weekly calibration runs using three ${ }^{228} \mathrm{Th}$ sources. The estimated FWHM energy resolution at the $Q_{\beta \beta}$ is $4.8 \pm 0.2 \mathrm{keV}$ for semi-coaxial and $3.2 \pm 0.2 \mathrm{keV}$ for BEGe detectors. The gain fluctuations between two subsequent calibrations were below the $0.05 \%$ (about $1 \mathrm{keV}$ at $Q_{\beta \beta}$ ), well below the energy resolution of the detectors.

In order to avoid biases in the $0 v \beta \beta$ decay analysis, for example in the data selection or in the optimization of the PSD parameters, all events with energy reconstructed within $20 \mathrm{keV}$ from the $Q_{\beta \beta}$ were automatically stored in a protected area during the data taking. Before the analysis has been finalized the collaboration had no access to these events. Their energy distribution, waveforms and number were unknown.

The spectrum of GERDA Phase I shows a few clear features (Fig. 3). In the low energy region $(<565 \mathrm{keV})$ the $\beta$-spectrum of ${ }^{39} \mathrm{Ar}$ is visible. Besides two prominent $\gamma$ lines from ${ }^{40} \mathrm{~K}(1460 \mathrm{keV})$ and ${ }^{42} K(1525 \mathrm{keV})$, the range from 500 to $1800 \mathrm{keV}$ is dominated by the $2 v \beta \beta$ spectrum. A few counts at higher energy $(1.7-2.6 \mathrm{MeV})$ can be related to different $\gamma$-lines from ${ }^{226} \mathrm{Ra}$ and ${ }^{228} \mathrm{Th}$ decay chains. In the high energy region $\left(>3 \mathrm{MeV}\right.$ ) mainly $\alpha$ from ${ }^{210} \mathrm{Po}$ and ${ }^{226} \mathrm{Ra}$ contribute to the spectrum. No background peaks were expected in the region of interest (ROI), where the main contributions are degraded $\alpha$ 's, Compton continuum and (especially for BEGe) betas from ${ }^{42} \mathrm{~K}$ decay on the crystals' surface. A detailed analysis of the background of the three data set (Golden, Silver and BEGe) is reported in [8].

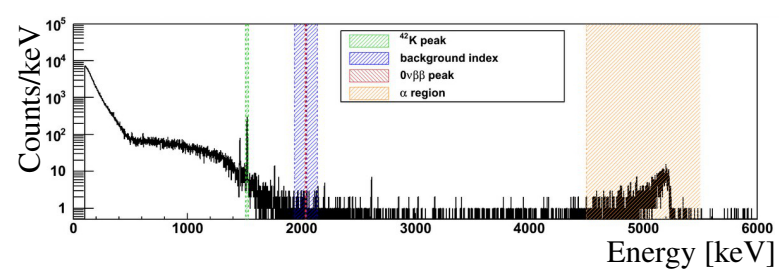

Figure 3: GERDA Phase I spectrum.

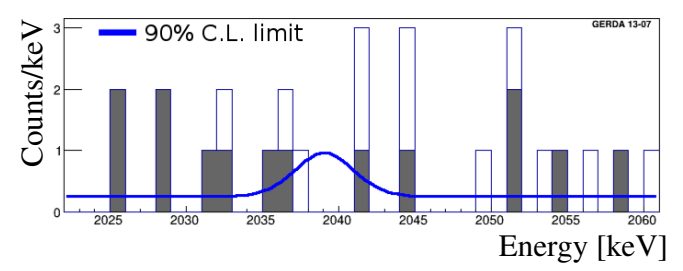

Figure 4: GERDA Phase I ROI spectrum, beforePSD (blue-empty) and after-PSD (gray-full).

\subsection{Phase I results}

Before the first unblinding GERDA collected $21.6 \mathrm{~kg} \cdot \mathrm{yr}$ of exposure with $62 \%$ (66\%) effi- 


\begin{tabular}{cc}
\hline $\mathrm{n}$ & $T_{1 / 2}^{0 v \chi}(90 \%$ C.I. $)$ \\
\hline 1 & $>4.2 \cdot 10^{23} \mathrm{yr}$ \\
2 & $>0.8 \cdot 10^{23} \mathrm{yr}$ \\
3 & $>1.8 \cdot 10^{23} \mathrm{yr}$ \\
7 & $>0.3 \cdot 10^{23} \mathrm{yr}$ \\
\hline
\end{tabular}

Table 1: Limits on $T^{0 v \chi}$.

\begin{tabular}{cc}
\hline Decay Mode & $T_{1 / 2}(90 \%$ C.L. $)$ \\
\hline $0_{\text {g.s. }}^{+}-2_{1}^{+}$ & $>1.6 \cdot 10^{23} \mathrm{yr}$ \\
$0_{\text {g.s. }}^{+}-0_{1}^{+}$ & $>3.7 \cdot 10^{23} \mathrm{yr}$ \\
$0_{\text {g.s. }}^{+}-2_{2}^{+}$ & $>2.3 \cdot 10^{23} \mathrm{yr}$ \\
\hline
\end{tabular}

Table 2: Limits on $2 v \beta \beta$ on excited states.

ciency for the semi-coaxial (BEGe) detectors. The efficiencies account for the isotopic abundance of ${ }^{76} \mathrm{Ge}$, the active volume of the crystals, the detection efficiency and the PSD signal acceptance. The median sensitivity was $2.4 \cdot 10^{25} \mathrm{yr}$.

The counts in the ROI (Fig. 4) were compatible with the background expectations of a flat distribution in the region from $1930 \mathrm{keV}$ to $2160 \mathrm{keV}$, the known $\gamma$ lines at $2014 \mathrm{keV}$ and $2119 \mathrm{keV}$ have been excluded from the fit. No counts were found within one $\sigma$ from the $Q_{\beta \beta}$. The profile likelihood fit of the three data set found a best value of zero signal counts $\left(N^{0 v}=0\right)$, and an upper limit of $N^{0 v}<3.5$ (90\% C.L) which correspond to:

$$
\begin{aligned}
T^{0 v} & >2.1 \cdot 10^{25} \mathrm{yr} \\
m_{e e} & >0.2-0.4 \mathrm{eV}
\end{aligned}
$$

The limit on the effective Majorana mass is computed using the recently recalculated phasespace factor for ${ }^{76} \mathrm{Ge}$ [9] and the nuclear matrix element (NME) calculations from [10, 11, 12, 13, 14, 15, 16]. More details about the analysis can be found in [17].

The GERDA experimented provided also the most precise $(\sigma<5 \%)$ measurement of ${ }^{76} \mathrm{Ge}$ $2 v \beta \beta$ half-life (3.3). This has been possible thanks to a signal to background ratio of 3:1 (4:1) in interval from 570-2039 (600-1800) keV. The analysis, described in [18], is based only on the golden data set

$$
T^{2 v}=1.926\left({ }_{-0.022}^{+0.025} \pm 0.092\right) \cdot 10^{21} \mathrm{yr} .
$$

The possibility of Majoron-emitting $\beta \beta$ decays has been investigated using the golden and the BEGe data set $(20.3 \mathrm{~kg} \cdot \mathrm{yr})$. The four possible spectral indices $(n=1,2,3,7)$ have been considered. No indication for a contribution of $0 v \beta \beta \chi$ decay was found. The lower limits extracted from the $90 \%$ probability (Table 1 ) of the marginalized posterior probability distribution are currently the most stringent on $T^{0 v \chi}$ for ${ }^{76} \mathrm{Ge}$. Details on these analyses can be found in [18].

The modularity of the GERDA array allows the search for $2 v \beta \beta$ to excited states transitions [19]. The signal of these processes would be the detection of de-excitation $\gamma$ rays from ${ }^{76} \mathrm{Se}$ in coincidence with the energy of the two $\mathrm{e}^{-}$in the detector where the $2 v \beta \beta$ occurred. Three decay modes have been considered: $0_{g . s}^{+}-2_{1}^{+} ; 0_{g . s}^{+}-0_{1}^{+} ; 0_{g . s}^{+}-2_{2}^{+}$. No signals were found and new half-life limits (Table 2), at least two orders of magnitude better than the previous, have been set. These results ruled out many NME calculations. 


\section{Upgrade to Phase II}

At the end of 2015 the data taking of GERDA Phase II started. The goal of this phase is to raise the sensitivity of the experiment above $10^{26} \mathrm{yr}$, one order of magnitude higher than in Phase I. To reach this goal we must at the same time increase the target mass, to collect exposure faster, and reduce the specific background. In particular to collect about $100 \mathrm{~kg} \cdot \mathrm{yr}$ of exposure in 3 to 4 years, 30 to $40 \mathrm{~kg}$ of detectors are needed. The background in the ROI must be reduced about 10 times to the level of $10^{-3} \mathrm{cts} /(\mathrm{keV} \cdot \mathrm{kg} \cdot \mathrm{yr})$.

In order to increase the target mass of Phase II to $\sim 36 \mathrm{~kg}$, the GERDA collaboration produced 30 BEGe detectors. The GERDA BEGe are based on a commercial model of Canberra, which has been customized for the needs of the $0 v \beta \beta$ search and to maximize the yield of enriched material during the diodes production. These detectors, described in Fig. 2 can provide energy resolution of about $3 \mathrm{keV}$ FWHM at the $Q_{\beta}$ when operated in the GERDA set-up.

The BEGe detectors also allowed better PSA performances with respect to the semi-coaxial. Since single site events from the bulk volume of the diodes (SSE) produce very similar pulses it is relatively easy to select this class of events. Simulations [20] and proxies (i.e. double escape peaks) shows that most of the signals $(\sim 90 \%)$ from $0 v \beta \beta$ are bulk SSE [6]. Other classes are associated to different topologies of the energy deposited in the detector and are more likely to arise from background events. Some examples of the different BEGe signals are shown in Fig. 5. Besides the SSE it is possible to distinguish multiple site events related to simultaneous energy depositions (i.e. $\gamma$ 's Compton scattering); $\mathrm{p}+$ contact pulses from interactions in the proximity of the read out contact (where $\alpha$ can reach the active volume); and $\mathrm{n}+$ surface pulses with slower rise-time for the increased charge collection time from the $\mathrm{p}-\mathrm{n}$ junction region (typical of signals from external $\beta$ emitters).
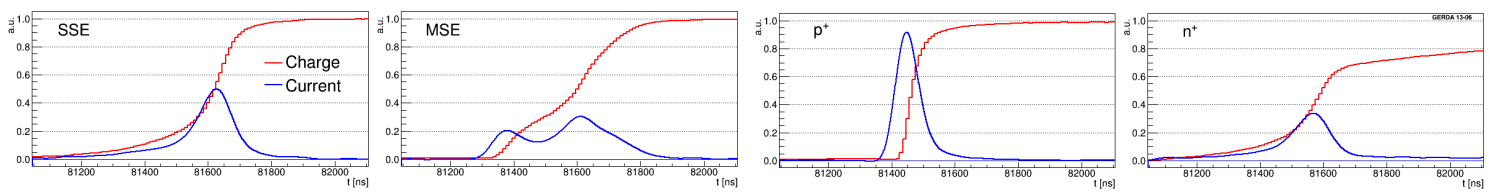

Figure 5: Examples of signals from BEGe detectors.

Several technical upgrades have been performed on the array. The number of strings has been increased from 4 to 7 to host the additional mass (Fig. 6). The amount of copper per $\mathrm{kg}$ of Ge has been reduced thanks to the insertion of mono-crystalline Si plates between the detectors (Fig. 7). Moreover to reduce the mechanical requirements of the holders and the material in the proximity of the detectors a novel contacting technique has been developed. In GERDA Phase II indeed all the detectors are connected to the high voltage and signal cables through thin bonded Al wires [21]. Each of the new strings can host 8 BEGe or 3 semi-coaxial detectors and is enclosed in a Nylon shroud (mini-shroud) to avoid the collection of ${ }^{42} \mathrm{~K}$ ions on the detectors' surface.

In Phase II GERDA is also taking advantage of the scintillation of liquid Ar to veto events not fully contained in the HPGe detector. The LAr is not fully transparent to its own scintillation light (attenuation length $\sim 50 \mathrm{~cm}$ ), hence wavelength-shifters to the visible range are needed. The visible light is then detected by PMT or SiPM. The shroud surrounding the array ( $\varnothing \sim 500 \mathrm{~mm})$ 

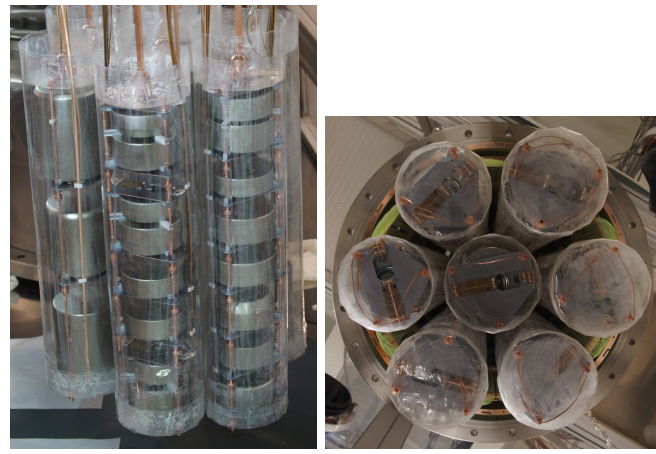

Figure 6: Detectors' array and mini-shrouds.

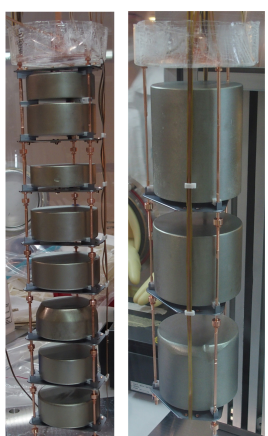

Figure 7: BEGe and semi-coaxial strings.

hosts the light detectors and is divided in three sections (Fig. 8). The top and the bottom sections are made of a thin copper foil coated with wavelength-shifter, these sections also host the 16 PMTs about $1 \mathrm{~m}$ away from the detector array. In the central session of the shroud a curtain of wavelength-shifting fibers can collect light coming from the inner volume or from the outer nearby volume and guide it toward silicon-photomultipliers (SiPM) placed at the end of the fibers. Also transparent mini-shrouds enclosing each string are coated with wavelength-shifter (Fig. 6).

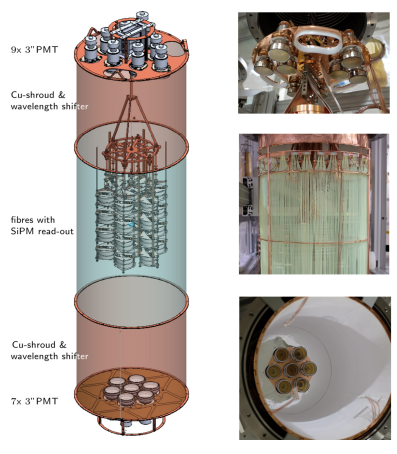

Figure 8: LAr scintillation veto system.

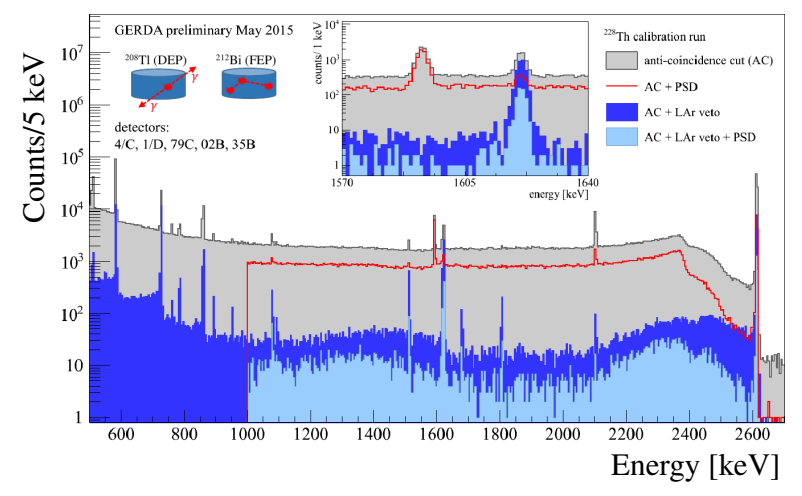

Figure 9: ${ }^{228}$ Th spectrum with a single string array.

During the commissioning of Phase II to test the performances of the LAr veto, a single BEGe string has been irradiated with a low intensity ${ }^{228} \mathrm{Th}$ source. The ${ }^{228} \mathrm{Th}$ besides being the isotope used for the periodic energy calibrations is also one of the most important background sources for GERDA. Fig. 9 shows the suppression that can be achieved for this kind of background through the combination of BEGe-PSA and LAr-veto. As can be seen in the two lines around 1.6 MeV, the two active background suppression techniques are complementary. For example the ${ }^{208} \mathrm{Tl}$ double escape peak at $1592 \mathrm{keV}$ is strongly suppressed by the LAr-veto because of the two $511 \mathrm{keV} \gamma$ 's escaping the Ge detector, while is not reduced by the PSD since the signals produced by a $1.5 \mathrm{MeV}$ pair $e^{-} e^{+}$are similar to the ones produced by a $2.039 \mathrm{MeV} e^{-} e^{-}$pair produced in the $0 v \beta \beta$.

\section{Phase II first data}

Since $20^{\text {th }}$ December 2015 Gerda Phase II is taking data in blind mode. The blinding is based on the same concept successfully adopted for Phase I. The energy of each event is recon- 
structed in real time by the DAQ and the events falling in a $50 \mathrm{keV}$ window around the ROI are stored in a protected area. The size of the blinded window is larger than the ROI $\left(Q_{\beta \beta} \pm 2 \sigma\right)$ to include possible gain instabilities and differences between the on-line filter and the off-line energy reconstruction used for the physics analysis. All 40 detectors are connected and active, see Table 3. About $2.6 \mathrm{~kg} \cdot \mathrm{yr}$ of exposure has been collected until the $11^{\text {th }}$ February 2016. The energy resolution in the calibrations is $\sim 3.4 \mathrm{keV}$ and $\sim 4.0 \mathrm{keV}$, FWHM at the ${ }^{208} \mathrm{Tl} 2.6 \mathrm{MeV}$ peak, for BEGe and semi-coaxial detectors respectively.

\begin{tabular}{cccc}
\hline $\mathrm{n}$ & type & & mass \\
\hline 30 & BEGe & enr. & $20.0 \mathrm{~kg}$ \\
7 & semi-coaxial & enr. & $15.8 \mathrm{~kg}$ \\
3 & semi-coaxial & nat. & $7.6 \mathrm{~kg}$ \\
\hline
\end{tabular}

Table 3: GERDA Phase II detectors.

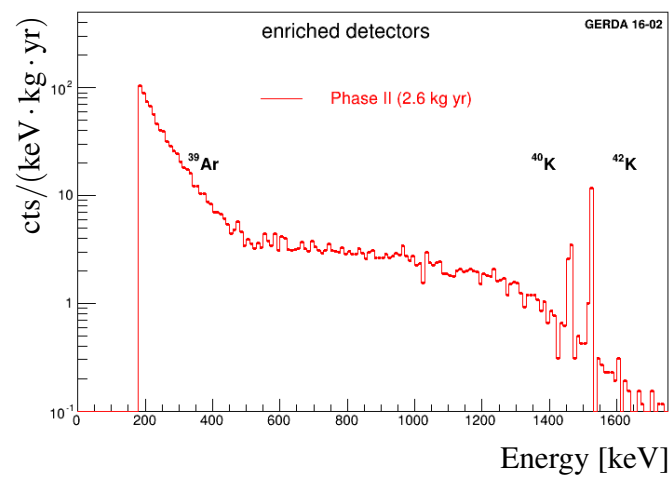

Figure 10: Phase II spectrum $(2.6 \mathrm{~kg} \cdot \mathrm{yr})$.

The energy spectrum of the two data set of enriched semi-coaxial and BEGe is shown in Fig. 10 after muon-veto and HPGe detectors anti-coincidence cut. It does not show any remarkable difference from the expectations from Phase I. The same main features are visible: ${ }^{39} \mathrm{Ar}$ beta spectrum, $2 v \beta \beta,{ }^{40} \mathrm{~K}$ and ${ }^{42} \mathrm{~K}$ lines.

The effect of the LAr-veto can be seen in Fig. 11. The ${ }^{40} \mathrm{~K} 1.46 \mathrm{MeV}$ electron capture is not associated to any coincidence energy deposition and shows therefore the signal acceptance of the veto $(98 \pm 4 \%)$. The ${ }^{42} \mathrm{~K} 1.52 \mathrm{MeV} \gamma$ instead follows a beta decay in LAr and is therefore strongly suppressed $(19 \pm 4 \%)$.

The $0 v \beta \beta$ signals would mostly $(\sim 90 \%)$ sit on the SSE band with a value of A/E normalized to 1 . The events with an $\mathrm{A} / \mathrm{E}$ value greater than 1 are associated with interactions in the $\mathrm{p}+$ contact region; here we can see the $\alpha$ background (mostly not vetoed). The points with values lower than 1 are mostly associated to multiple site events from $\gamma$, these are visible in the two $K$ lines and in their Compton continuum regions. Below the SSE band sit also the events from the $\mathrm{n}+$ surface (e.g. $\beta$ from ${ }^{42} \mathrm{~K}$ ) but this component is still not clearly visible in the data.

\section{Conclusion}

The GERDA experiment is leading the $0 v \beta \beta$ search with ${ }^{76} \mathrm{Ge}$, its Phase I provided the best limit for $T_{76}^{0 v \beta \beta}>2.1 \cdot 10^{25} \mathrm{yr}\left(90 \%\right.$ C.L.) and one of the best limits on $m_{e e}>0.2-0.4 \mathrm{eV}$ ( $90 \%$ C.L.). From the lower energy part of the spectrum $(0.5-2 \mathrm{MeV})$ the GERDA collaboration provides the best measurement of ${ }^{76} \mathrm{Ge} 2 v \beta \beta$ half-life and new limits on $0 v \beta \beta$ with Majorons emission and on the $2 v \beta \beta$ on ${ }^{76} \mathrm{Se}$ excited states. Since the end of 2015 GERDA is running with an improved setup (Phase II). The higher target mass (35 kg of enriched Ge) of the new array 


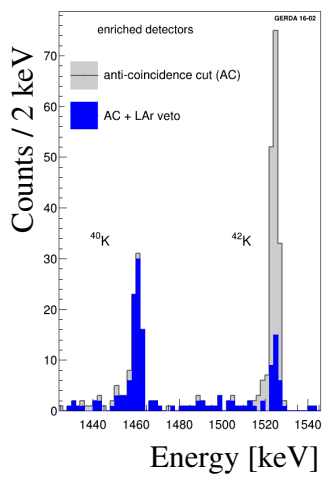

Figure 11: LAr-veto on $\mathrm{K}$ lines.

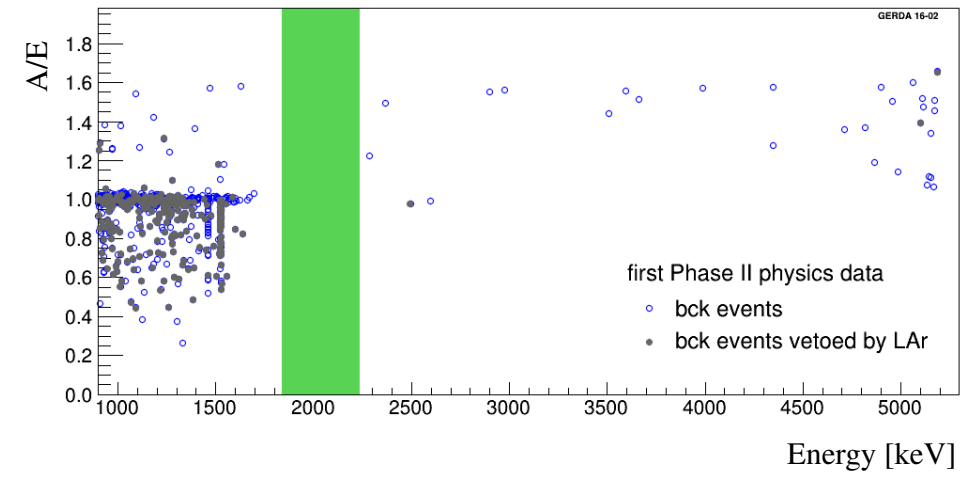

Figure 12: PSD classifier for BEGe detectors.

( 37 enr. and 3 nat. HPGe) will allow to quickly double the exposure of Phase I and reach the goal of $100 \mathrm{~kg} \cdot \mathrm{yr}$ in a few years of data-taking. The background index goal of $10^{-3} \mathrm{cts} /(\mathrm{keV} \cdot \mathrm{kg} \cdot \mathrm{yr})$ will be reached thanks to the new LAr scintillation light veto system and the enhanced pulse shape discrimination performance of the 30 BEGe detectors. In the first $\sim 2$ months of data taking an exposure of $2.6 \mathrm{~kg} \cdot \mathrm{yr}$ has been collected and no unforeseen background components appear to be present. 


\section{References}

[1] M. Goeppert Mayer, Double beta-disintegration, Phys. Rev. 48 (1935) 512-516.

[2] V. I. Tretyak and Y. G. Zdesenko, Tables of double beta decay - an update At. Data Nucl. Data Tables 80,83 (2002).

[3] J. Schechter, J.W.F. Valle, Neutrinoless double- $\beta$ decay in $S U(2) X U(1)$ theories, Phys. Rev. D 25 (1982) 2951.

[4] M. Agostini et al. (GERDA Collaboration), The GERDA Experiment for the search of $0 v \beta \beta$ decay in ${ }^{76}$ Ge, Eur. Phys. J. C 73 (2013) 2330.

[5] D. Budjáš et al., Pulse shape discrimination studies with a Broad-Energy Germanium detector for signal identification and background suppression in the GERDA double beta decay experiment, JINST 4 (2009) P10007.

[6] M. Agostini et al. (GERDA Collaboration), Pulse shape discrimination for GERDA Phase I data, Eur. Phys. J. C 73 (2013) 2583

[7] M. Agostini, Signal and background studies for the search of neutrinoless double beta decay in GERDA, PhD thesis, TU München, 2013, chapter 6.

[8] M. Agostini et al. (GERDA Collaboration), The background of the $0 v \beta \beta$ experiment GERDA, Eur. Phys. J. C 74 (2014) 2764.

[9] J. Kotila ant F. Iachello, Phase-space factors for double- $\beta$ decay, Phys. Rev. C 85 (2012) 034316.

[10] T. R. Rodríguez and G. Martínez-Pinedo, Phys. Rev. Lett. 105 (2010) 252503.

[11] J. Menéndez et al., Nucl. Phys. A 818 (2009) 139-151.

[12] J. Barea et al., Phys. Rev. C 87 (2013) 014315.

[13] J. Suhonen ant O. Civitarese, Nucl. Phys. A 847 (2010) 207-232.

[14] A. Meroni et al., JHEP 1302 (2013) 25.

[15] F. Simkovic et al., Phys. Rev. C 87 (2013) 045501.

[16] M. T. Mustonen and J. Engel, Phys. Rev. C 87 (2013) 064302.

[17] M. Agostini et al. (GERDA Collaboration), Results on Neutrinoless Double- $\beta$ Decay of ${ }^{76}$ Ge from Phase I of the GERDA Experiment, Phys. Rev. Lett. 111 (2013) 122503.

[18] M. Agostini et al. (GERDA Collaboration), Results on $\beta \beta$ decay with emission of two neutrinos or Majorons in ${ }^{76} \mathrm{Ge}$ from GERDA Phase I, Eur. Phys. J. C 75 (2015) 416.

[19] M. Agostini et al. (GERDA Collaboration), $2 v \beta \beta$ decay of ${ }^{76}$ Ge into excited states with GERDA Phase I, J. Phys. G42 (2015) 115201.

[20] M. Agostini et al., Signal modeling of high-purity Ge detectors with a small read-out electrode and application to neutrinoless double beta decay search in Ge-76, JINST, 6 (2011) P03005

[21] T. Bode, The neutrinoless double beta decay experiment GERDA Phase II: A novel ultra-low background contacting technique for germanium detectors and first background data, $\mathrm{PhD}$ thesis, TU München, 2016. 